\title{
Some new classes of generalized Lagrange-based Apostol type Hermite polynomials
}

\section{Waseem A. Khan ${ }^{1}$ and Nisar $\mathrm{K} \mathrm{S}^{2}$}

${ }^{1}$ Department of Mathematics, Faculty of Science, Integral University, Lucknow-226026, India

${ }^{2}$ Department of Mathematics, College of Arts and Science-Wadi Al dawaser, Prince Sattam bin Abdulaziz University, Riyadh region 11991, Saudi Arabia

E-mail: waseem08_khan@rediffmail.com,n.sooppy@psau.edu.sa

\begin{abstract}
In this paper, we introduce a general family of Lagrange-based Apostoltype Hermite polynomials thereby unifying the Lagrange-based Apostol HermiteBernoulli and the Lagrange-based Apostol Hermite-Genocchi polynomials. We also define Lagrange-based Apostol Hermite-Euler polynomials via the generating function. In terms of these generalizations, we find new and useful relations between the unified family and the Apostol Hermite-Euler polynomials. We also derive their explicit representations and list some basic properties of each of them. Some implicit summation formulae and general symmetry identities are derived by using different analytical means and applying generating functions.
\end{abstract}

2010 Mathematics Subject Classification: 11B68, 11B65, 33C45.

Keywords: Hermite polynomials, Chan-Chyan-Srivastava polynomials, Lagrangebased Apostol type Hermite polynomials, summation formulae, symmetric identities.

\section{Introduction}

The Lagrange polynomials in several variables, which are known as the ChanChyan-Srivastava polynomials [3] are defined by means of the following generating function:

$$
\begin{gathered}
\prod_{j=1}^{r}\left(1-x_{j} t\right)^{-\alpha_{j}}=\sum_{n=0}^{\infty} g_{n}^{\left(\alpha_{1} \ldots \alpha_{r}\right)}\left(x_{1}, \ldots ., x_{r}\right) t^{n} \\
\left(\alpha_{j} \in \mathbb{C}(j=1, \ldots, r) ;|t|<\min \left\{\left|x_{1}\right|^{-1}, \ldots,\left|x_{r}\right|^{-1}\right\}\right)
\end{gathered}
$$

and are given explicitly by

$$
\begin{gathered}
g_{n}^{\left(\alpha_{1}, \alpha_{2} \ldots \alpha_{r}\right)}\left(x_{1}, x_{2}, \ldots, x_{r}\right)=\sum_{k_{r-1}=0}^{n} \ldots \sum_{k_{2}}^{k_{3}} \sum^{k_{2}}\left(\alpha_{1}\right)_{k_{1}}\left(\alpha_{2}\right)_{k_{2}-k_{1} \ldots\left(\alpha_{r-1}\right)_{k_{r-1}-k_{r-2}}\left(\alpha_{r}\right)_{n-k_{r-1}}} \\
\cdot \frac{x_{1}^{k_{1}}}{k_{1} !} \frac{x_{2}^{k_{2}-k_{1}}}{\left(k_{2}-k_{1}\right) !} \ldots \frac{x_{r-1}^{k_{r-1}-k_{r-2}}}{\left(k_{r-1}-k_{r-2}\right) !} \frac{x_{r}^{n-k_{r}-1}}{\left(n-k_{r}-1\right) !}
\end{gathered}
$$

where and in what follows $(\lambda)_{n}$ denotes the Pochhamer symbol (or the shifted factorial) defined by

$$
(\lambda)_{0}:=1 \text { and }(\lambda)_{n}=\lambda(\lambda) \ldots(\lambda+n-1)(n \in \mathbb{N}:=\{1,2,3, \ldots\}) .
$$

Luo and Srivastava $[10,11,12]$ introduced the generalized Apostol-Bernoulli polynomials $B_{n}^{(\alpha)}(x)$ of order $\alpha$, Luo [7] investigated the generalized Apostol-Euler polynomials $E_{n}^{(\alpha)}(x)$ of order $\alpha$ and the generalized Apostol-Genocchi polynomials $G_{n}^{(\alpha)}(x)$ of order $\alpha$ (see also $[6,8,9]$ ).

The generalized Apostol-Bernoulli polynomials $B_{n}^{(\alpha)}(x ; \lambda)$ of order $\alpha \in \mathbb{C}$, the generalized Apostol-Euler polynomials $E_{n}^{(\alpha)}(x ; \lambda)$ of order $\alpha \in \mathbb{C}$, the generalized 
Apostol-Genocchi polynomials $G_{n}^{(\alpha)}(x ; \lambda)$ of order $\alpha \in \mathbb{C}$ are defined respectively by the following generating functions:

$$
\begin{aligned}
& \left(\frac{t}{\lambda e^{t}-1}\right)^{\alpha} e^{x t}=\sum_{n=0}^{\infty} B_{n}^{(\alpha)}(x ; \lambda) \frac{t^{n}}{n !},\left(|t|<|\ln \lambda|<2 \pi ; 1^{\alpha}=1\right) \\
& \left(\frac{2}{\lambda e^{t}-1}\right)^{\alpha} e^{x t}=\sum_{n=0}^{\infty} E_{n}^{(\alpha)}(x ; \lambda) \frac{t^{n}}{n !},\left(|t|<|\ln \lambda|<\pi ; 1^{\alpha}=1\right)
\end{aligned}
$$

and

$$
\left(\frac{2 t}{\lambda e^{t}-1}\right)^{\alpha} e^{x t}=\sum_{n=0}^{\infty} G_{n}^{(\alpha)}(x ; \lambda) \frac{t^{n}}{n !},\left(|t|<|\ln \lambda|<\pi ; 1^{\alpha}=1\right) .
$$

It is easy to see that

$$
B_{n}^{(\alpha)}(x)=B_{n}^{(\alpha)}(x ; 1), E_{n}^{(\alpha)}(x)=E_{n}^{(\alpha)}(x ; 1) \text { and } G_{n}^{(\alpha)}(x)=G_{n}^{(\alpha)}(x ; 1) .
$$

Recently, Srivastava et al. [16] introduce and investigate the following class of Lagrange-based Apostol type polynomials $T_{n . \lambda, k}^{\left(\alpha_{1}, \cdots, \alpha_{r} ; \alpha\right)}\left(x_{1} \cdots x_{r} ; x\right)$ as follows:

Definition 1.1. The Lagrange-based Apostol type polynomials $T_{n, \lambda, k}^{\left(\alpha_{1}, \cdots, \alpha_{r} ; \alpha\right)}\left(x_{1} \ldots\right.$ $\left.x_{r} ; x\right)$ are defined by means of the following generating function:

$$
\begin{gathered}
\prod_{j=1}^{r}\left(1-x_{j} t\right)^{-\alpha_{j}}\left(\frac{2^{k} t}{\lambda e^{t}+(-1)^{k+1}}\right)^{\alpha} e^{x t}=\sum_{n=0}^{\infty} T_{n, \lambda, k}^{\left(\alpha_{1}, \cdots, \alpha_{r} ; \alpha\right)}\left(x_{1} \cdots x_{r} ; x\right) t^{n} \\
\left(\lambda \in \mathbb{C} ; \alpha_{j} \in \mathbb{C}(j=1, \cdots, r)\right) .
\end{gathered}
$$

In the particular cases when $k=0$ and $k=1$, we define the Lagrange-based Apostol-Bernoulli polynomials $B_{n, \lambda}^{\left(\alpha_{1}, \cdots, \alpha_{r} ; \alpha\right)}\left(x_{1} \cdots x_{r} ; x\right)$ and the Lagrange-based ApostolGenocchi polynomials $G_{n, \lambda}^{\left(\alpha_{1}, \cdots, \alpha_{r} ; \alpha\right)}\left(x_{1} \cdots x_{r} ; x\right)$ as follows.

Definition 1.2. The Lagrange-based Apostol-Bernoulli polynomials $B_{n, \lambda}^{\left(\alpha_{1}, \cdots, \alpha_{r} ; \alpha\right)}\left(x_{1}\right.$. $\left.\cdots x_{r} ; x\right)$ given by

$$
B_{n, \lambda}^{\left(\alpha_{1}, \cdots, \alpha_{r} ; \alpha\right)}\left(x_{1} \cdots x_{r} ; x\right)=T_{n, \lambda, 0}^{\left(\alpha_{1}, \cdots, \alpha_{r} ; \alpha\right)}\left(x_{1} \cdots x_{r} ; x\right)
$$

are defined by means of the following generating function

$$
\begin{gathered}
\prod_{j=1}^{r}\left(1-x_{j} t\right)^{-\alpha_{j}}\left(\frac{t}{\lambda e^{t}-1}\right)^{\alpha} e^{x t}=\sum_{n=0}^{\infty} B_{n, \lambda}^{\left(\alpha_{1}, \cdots, \alpha_{r} ; \alpha\right)}\left(x_{1} \cdots x_{r} ; x\right) t^{n}, \\
\left(\lambda \in \mathbb{C} ; \alpha_{j} \in \mathbb{C}(j=1, \cdots, r)\right) .
\end{gathered}
$$

Definition 1.3. The Lagrange-based Apostol-Genocchi polynomials $G_{n, \lambda}^{\left(\alpha_{1}, \cdots, \alpha_{r} ; \alpha\right)}\left(x_{1}\right.$. $\left.\cdots x_{r} ; x\right)$ given by

$$
G_{n, \lambda}^{\left(\alpha_{1}, \cdots, \alpha_{r} ; \alpha\right)}\left(x_{1} \cdots x_{r} ; x\right)=T_{n, \lambda, 1}^{\left(\alpha_{1}, \cdots, \alpha_{r} ; \alpha\right)}\left(x_{1} \cdots x_{r} ; x\right)
$$

are defined by means of the following generating function

$$
\begin{gathered}
\prod_{j=1}^{r}\left(1-x_{j} t\right)^{-\alpha_{j}}\left(\frac{2 t}{\lambda e^{t}+1}\right)^{\alpha} e^{x t}=\sum_{n=0}^{\infty} G_{n, \lambda}^{\left(\alpha_{1}, \cdots, \alpha_{r} ; \alpha\right)}\left(x_{1} \cdots x_{r} ; x\right) t^{n}, \\
\left(\lambda \in \mathbb{C} ; \alpha_{j} \in \mathbb{C}(j=1, \cdots, r)\right) .
\end{gathered}
$$
lows.

Furthermore, we define the Lagrange-based Apostol-Euler polynomials as fol- 
Definition 1.4. The Lagrange-based Apostol-Euler polynomials $E_{n, \lambda}^{\left(\alpha_{1}, \cdots, \alpha_{r} ; \alpha\right)}\left(x_{1} \cdots\right.$ $\left.x_{r} ; x\right)$ are defined by means of the following generating function:

$$
\begin{gathered}
\prod_{j=1}^{r}\left(1-x_{j} t\right)^{-\alpha_{j}}\left(\frac{2}{\lambda e^{t}+1}\right)^{\alpha} e^{x t}=\sum_{n=0}^{\infty} E_{n, \lambda}^{\left(\alpha_{1}, \cdots, \alpha_{r} ; \alpha\right)}\left(x_{1} \cdots x_{r} ; x\right) t^{n}, \\
\left(\lambda \in \mathbb{C} ; \alpha_{j} \in \mathbb{C}(j=1, \cdots, r)\right) .
\end{gathered}
$$

The 2-variable Hermite Kampé de Fériet polynomials $(2 \mathrm{VHKdFP}) H_{n}(x, y)[2$, 4] are defined as

$$
e^{x t+y t^{2}}=\sum_{n=0}^{\infty} H_{n}(x, y) \frac{t^{n}}{n !}
$$

are solutions of the heat equation

$$
\begin{gathered}
\frac{\partial}{\partial y} H_{n}(x, y)=\frac{\partial^{2}}{\partial x^{2}} H_{n}(x, y), \\
H_{n}(x, 0)=x^{n} .
\end{gathered}
$$

The higher order Hermite polynomials, sometimes called the Kampe de Feriet polynomials of order $\mathrm{m}$ or the Gould Hopper polynomials (GHP) $H_{n}^{(m)}(x, y)$ defined by the generating function [1]

$$
e^{x t+y t^{m}}=\sum_{n=0}^{\infty} H_{n}^{(m)}(x, y) \frac{t^{n}}{n !}
$$

are solutions of the generalized heat equation [4]

$$
\begin{gathered}
\frac{\partial}{\partial y} f(x, y)=\frac{\partial^{m}}{\partial x^{m}} f(x, y) \\
f(x, 0)=x^{n} .
\end{gathered}
$$

Also we note that

$$
\begin{aligned}
& H_{n}^{(2)}(x, y)=H_{n}(x, y), \\
& H_{n}(2 x,-1)=H_{n}(x),
\end{aligned}
$$

where $H_{n}(x)$ are the classical Hermite polynomials [1].

The Lagrange-based generalizations which we have introduced above, enable us to obtain new and useful relations between the Apostol-Bernoulli polynomials, Apostol-Euler polynomials and the Apostol-Genocchi polynomials. In this paper, we first study several elementary properties of the generalized Lagrange-based Apostol type Hermite polynomials $T_{n, \lambda, k}^{\left(\alpha_{1}, \cdots, \alpha_{r} ; \alpha\right)}\left(x_{1} \cdots x_{r} ; x, y\right)$. Some implicit summation formulae and general symmetry identities are derived by using different analytical means and applying generating functions.

\section{Definitions and Basic Properties of the Generalized Lagrange-Based Apostol-Type Polynomials $T_{n, \lambda, k}^{\left(\alpha_{1}, \cdots, \alpha_{r} ; \alpha\right)}\left(x_{1} \cdots x_{r} ; x, y\right)$}

In this section, we introduce Lagrange-based Apostol type Hermite polynomials and give the explicit representations and list basic properties of the generalized Lagrange-based Apostol-type Hermite polynomials $T_{n, \lambda, k}^{\left(\alpha_{1}, \cdots, \alpha_{r} ; \alpha\right)}\left(x_{1} \cdots x_{r} ; x, y\right)$. 
Definition 2.1. The Lagrange-based Apostol type Hermite polynomials $T_{n, \lambda, k}^{\left(\alpha_{1}, \cdots, \alpha_{r} ; \alpha\right)}\left(x_{1}\right.$. $\left.\cdots x_{r} ; x, y\right)$ are defined by means of the following generating function:

$$
\begin{gathered}
\prod_{j=1}^{r}\left(1-x_{j} t\right)^{-\alpha_{j}}\left(\frac{2^{k} t}{\lambda e^{t}+(-1)^{k+1}}\right)^{\alpha} e^{x t+y t^{2}}=\sum_{n=0}^{\infty} T_{n, \lambda, k}^{\left(\alpha_{1}, \cdots, \alpha_{r} ; \alpha\right)}\left(x_{1} \cdots x_{r} ; x, y\right) t^{n}, \\
\left(\lambda \in \mathbb{C} ; \alpha_{j} \in \mathbb{C}(j=1, \cdots, r)\right) .
\end{gathered}
$$

In the particular cases, when $k=0$ and $k=1$, we define the Lagrange-based Apostol Hermite-Bernoulli polynomials ${ }_{H} B_{n, \lambda}^{\left(\alpha_{1}, \cdots, \alpha_{r} ; \alpha\right)}\left(x_{1} \cdots x_{r} ; x, y\right)$ and the Lagrangebased Apostol Hermite-Genocchi polynomials ${ }_{H} G_{n, \lambda}^{\left(\alpha_{1}, \cdots, \alpha_{r} ; \alpha\right)}\left(x_{1} \cdots x_{r} ; x, y\right)$ as follows.

Definition 2.2. The Lagrange-based Apostol Hermite-Bernoulli polynomials ${ }_{H} B_{n, \lambda}^{\left(\alpha_{1}, \cdots, \alpha_{r} ; \alpha\right)}\left(x_{1}\right.$. $\left.\cdots x_{r} ; x, y\right)$ are defined by means of the following generating function:

$$
\begin{gathered}
\prod_{j=1}^{r}\left(1-x_{j} t\right)^{-\alpha_{j}}\left(\frac{t}{\lambda e^{t}-1}\right)^{\alpha} e^{x t+y t^{2}}=\sum_{n=0}^{\infty} H_{n, \lambda}^{\left(\alpha_{1}, \cdots, \alpha_{r} ; \alpha\right)}\left(x_{1} \cdots x_{r} ; x, y\right) t^{n} \\
\left(\lambda \in \mathbb{C} ; \alpha_{j} \in \mathbb{C}(j=1, \cdots, r)\right) .
\end{gathered}
$$

Definition 2.3. The Lagrange-based Apostol Hermite-Genocchi polynomials ${ }_{H} G_{n, \lambda}^{\left(\alpha_{1}, \cdots, \alpha_{r} ; \alpha\right)}\left(x_{1}\right.$. $\left.\cdots x_{r} ; x, y\right)$ are defined by means of the following generating function:

$$
\begin{gathered}
\prod_{j=1}^{r}\left(1-x_{j} t\right)^{-\alpha_{j}}\left(\frac{2 t}{\lambda e^{t}+1}\right)^{\alpha} e^{x t+y t^{2}}=\sum_{n=0}^{\infty} H_{n, \lambda}^{\left(\alpha_{1}, \cdots, \alpha_{r} ; \alpha\right)}\left(x_{1} \cdots x_{r} ; x, y\right) t^{n} \\
\left(\lambda \in \mathbb{C} ; \alpha_{j} \in \mathbb{C}(j=1, \cdots, r)\right) .
\end{gathered}
$$

Furthermore, we define the Lagrange-based Apostol Hermite-Euler polynomials as follows.

Definition 2.4. The Lagrange-based Apostol Hermite-Euler polynomials ${ }_{H} E_{n, \lambda}^{\left(\alpha_{1}, \cdots, \alpha_{r} ; \alpha\right)}\left(x_{1}\right.$. $\left.\cdots x_{r} ; x, y\right)$ are defined by means of the following generating function:

$$
\begin{gathered}
\prod_{j=1}^{r}\left(1-x_{j} t\right)^{-\alpha_{j}}\left(\frac{2}{\lambda e^{t}+1}\right)^{\alpha} e^{x t+y t^{2}}=\sum_{n=0}^{\infty} H_{n, \lambda}^{\left(\alpha_{1}, \cdots, \alpha_{r} ; \alpha\right)}\left(x_{1} \cdots x_{r} ; x, y\right) t^{n} \\
\left(\lambda \in \mathbb{C} ; \alpha_{j} \in \mathbb{C}(j=1, \cdots, r)\right) .
\end{gathered}
$$

Theorem 2.1. Each of the following relationships holds true:

$$
\begin{gathered}
{ }_{H} B_{n, \lambda}^{\left(\alpha_{1}, \cdots, \alpha_{r} ; \alpha\right)}(0, \cdots, 0 ; x, y)=\frac{{ }_{H} B_{n}^{(\alpha)}}{n !}(x, y ; \lambda) \\
{ }_{H} E_{n, \lambda}^{\left(\alpha_{1}, \cdots, \alpha_{r} ; \alpha\right)}(0, \cdots, 0 ; x, y)=\frac{{ }_{H} E_{n}^{(\alpha)}}{n !}(x, y ; \lambda) \\
{ }_{H} G_{n, \lambda}^{\left(\alpha_{1}, \cdots, \alpha_{r} ; \alpha\right)}(0, \cdots, 0 ; x, y)=\frac{{ }_{H} G_{n}^{(\alpha)}}{n !}(x, y ; \lambda) \\
{ }_{H} B_{n, \lambda}^{\left(\alpha_{1}, \cdots, \alpha_{r} ; \alpha\right)}\left(x_{1}, \cdots, x_{r} ; x, 0\right)=\sum_{l=0}^{n} \frac{B_{n-l}^{\left(\alpha_{1}, \cdots, \alpha_{r} ; \alpha\right)}(x ; \lambda) g_{l}^{\left(\alpha_{1}, \cdots, \alpha_{r} ; \alpha\right)}\left(x_{1}, \cdots, x_{r}\right)}{(n-l) !} \\
{ }_{H} E_{n, \lambda}^{\left(\alpha_{1}, \cdots, \alpha_{r} ; \alpha\right)}\left(x_{1}, \cdots, x_{r} ; x, 0\right)=\sum_{l=0}^{n} \frac{E_{n-l}^{\left(\alpha_{1}, \cdots, \alpha_{r} ; \alpha\right)}(x ; \lambda) g_{l}^{\left(\alpha_{1}, \cdots, \alpha_{r} ; \alpha\right)}\left(x_{1}, \cdots, x_{r}\right)}{(n-l) !} \\
{ }_{H} G_{n, \lambda}^{\left(\alpha_{1}, \cdots, \alpha_{r} ; \alpha\right)}\left(x_{1}, \cdots, x_{r} ; x, 0\right)=\sum_{l=0}^{n} \frac{G_{n-l}^{\left(\alpha_{1}, \cdots, \alpha_{r} ; \alpha\right)}(x ; \lambda) g_{l}^{\left(\alpha_{1}, \cdots, \alpha_{r} ; \alpha\right)}\left(x_{1}, \cdots, x_{r}\right)}{(n-l) !}
\end{gathered}
$$




$$
\begin{gathered}
H B_{n, \lambda}^{\left(\alpha_{1}, \cdots, \alpha_{r} ; 1\right)}(0, \cdots, 0 ; 0,0)=B_{n}(\lambda) . \\
H E_{n, \lambda}^{\left(\alpha_{1}, \cdots, \alpha_{r} ; 1\right)}(0, \cdots, 0 ; 0,0)=E_{n}(\lambda) . \\
{ }_{H} G_{n, \lambda}^{\left(\alpha_{1}, \cdots, \alpha_{r} ; 1\right)}(0, \cdots, 0 ; 0,0)=G_{n}(\lambda) . \\
{ }_{H} B_{n, \lambda}^{\left(\alpha_{1}, \cdots, \alpha_{r} ; 0\right)}\left(x_{1}, \cdots, x_{r} ; 0,0\right)={ }_{H} E_{n, \lambda}^{\left(\alpha_{1}, \cdots, \alpha_{r} ; 0\right)}\left(x_{1}, \cdots, x_{r} ; 0,0\right) .
\end{gathered}
$$

and

$$
={ }_{H} G_{n, \lambda}^{\left(\alpha_{1}, \cdots, \alpha_{r} ; 0\right)}\left(x_{1}, \cdots, x_{r} ; 0,0\right)=g_{n}^{\left(\alpha_{1}, \cdots, \alpha_{r}\right)}\left(x_{1}, \cdots, x_{r}\right) .
$$

Theorem 2.2. The Lagrange-based Apostol Hermite Bernoulli polynomials, the Lagrange-based Apostol Hermite Euler polynomials and the Lagrange-based Apostol Hermite Genocchi polynomials are explicitly given by

$$
\begin{aligned}
& { }_{H} B_{n, \lambda}^{\left(\alpha_{1}, \cdots, \alpha_{r} ; \alpha\right)}\left(x_{1}, \cdots, x_{r} ; x, y\right)=\sum_{l=0}^{n} \frac{{ }_{H} B_{n-l}^{(\alpha)}(x, y ; \lambda) g_{l}^{\left(\alpha_{1}, \cdots, \alpha_{r}\right)}\left(x_{1}, \cdots, x_{r}\right)}{(n-l) !} . \\
& { }_{H} E_{n, \lambda}^{\left(\alpha_{1}, \cdots, \alpha_{r} ; \alpha\right)}\left(x_{1}, \cdots, x_{r} ; x, y\right)=\sum_{l=0}^{n} \frac{{ }_{H} E_{n-l}^{(\alpha)}(x, y ; \lambda) g_{l}^{\left(\alpha_{1}, \cdots, \alpha_{r}\right)}\left(x_{1}, \cdots, x_{r}\right)}{(n-l) !} .
\end{aligned}
$$

and

$$
{ }_{H} G_{n, \lambda}^{\left(\alpha_{1}, \cdots, \alpha_{r} ; \alpha\right)}\left(x_{1}, \cdots, x_{r} ; x, y\right)=\sum_{l=0}^{n} \frac{{ }_{H} G_{n-l}^{(\alpha)}(x, y ; \lambda) g_{l}^{\left(\alpha_{1}, \cdots, \alpha_{r}\right)}\left(x_{1}, \cdots, x_{r}\right)}{(n-l) !} .
$$

respectively.

Proof. It is fairly straightforward to observe from (1.1) and (2.2)

$$
\begin{gathered}
{ }_{H} B_{n, \lambda}^{\left(\alpha_{1}, \cdots, \alpha_{r} ; \alpha\right)}\left(x_{1}, \cdots, x_{r} ; x, y\right)=\left[\left(\frac{t}{\lambda e^{t}-1}\right)^{\alpha} e^{x t+y t^{2}}\right]\left(\prod_{j=1}^{r}\left(1-x_{j} t\right)^{-\alpha_{j}}\right) \\
=\left(\sum_{n=0}^{\infty} H_{n} B_{n}^{(\alpha)}(x, y ; \lambda) \frac{t^{n}}{n !}\right)\left(\sum_{l=0}^{\infty} g_{l}^{\left(\alpha_{1}, \cdots, \alpha_{r}\right)} t^{l}\right) \\
=\sum_{n=0}^{\infty}\left(\sum_{l=0}^{n} \frac{{ }_{H} B_{n-l}^{(\alpha)}(x, y ; \lambda) g_{l}^{\left(\alpha_{1}, \cdots, \alpha_{r}\right)}\left(x_{1}, \cdots, x_{r}\right)}{(n-l) !}\right) t^{n}
\end{gathered}
$$

Comparing the coefficient of $t^{n}$ in both sided, we get the result (2.16). The explicit representations of ${ }_{H} E_{n, \lambda}^{\left(\alpha_{1}, \cdots, \alpha_{r} ; \alpha\right)}\left(x_{1}, \cdots, x_{r} ; x, y\right)$ and ${ }_{H} G_{n, \lambda}^{\left(\alpha_{1}, \cdots, \alpha_{r} ; \alpha\right)}\left(x_{1}, \cdots, x_{r} ; x, y\right)$ follow in a similar manner from the generating function (1.1) in conjunction with (2.3) and (2.4) respectively.

Theorem 2.3. The following identities hold true for the Lagrange-based Apostol type Hermite-Bernoulli polynomials, the Lagrange-based Apostol type Hermite-Euler polynomials and the Lagrange-based Apostol type Hermite-Genocchi polynomials

$$
\begin{gathered}
T_{n, \lambda, k}^{\left(\alpha_{1}+\beta_{1}, \cdots, \alpha_{r}+\beta_{r} ; \alpha+\beta\right)}\left(x_{1} \cdots x_{r} ; x+z, y+u\right) \\
=\sum_{l=0}^{n} T_{l, \lambda, k}^{\left(\alpha_{1}, \cdots, \alpha_{r} ; \alpha\right)}\left(x_{1} \cdots x_{r} ; x, y\right) T_{n-l, \lambda, k}^{\left(\beta_{1}, \cdots, \beta_{r} ; \beta\right)}\left(x_{1} \cdots x_{r} ; z, u\right) . \\
{ }_{H} B_{n, \lambda}^{\left(\alpha_{1}+\beta_{1}, \cdots, \alpha_{r}+\beta_{r} ; \alpha+\beta\right)}\left(x_{1} \cdots x_{r} ; x+z, y+u\right) \\
\sum_{l=0}^{n}{ }_{H} B_{l, \lambda}^{\left(\alpha_{1}, \cdots, \alpha_{r} ; \alpha\right)}\left(x_{1} \cdots x_{r} ; x, y\right)_{H} B_{n-l, \lambda}^{\left(\beta_{1}, \cdots, \beta_{r} ; \beta\right)}\left(x_{1} \cdots x_{r} ; z, u\right) . \\
{ }_{H} E_{n, \lambda}^{\left(\alpha_{1}+\beta_{1}, \cdots, \alpha_{r}+\beta_{r} ; \alpha+\beta\right)}\left(x_{1} \cdots x_{r} ; x+z, y+u\right)
\end{gathered}
$$




$$
=\sum_{l=0}^{n}{ }_{H} E_{l, \lambda}^{\left(\alpha_{1}, \cdots, \alpha_{r} ; \alpha\right)}\left(x_{1} \cdots x_{r} ; x, y\right)_{H} E_{n-l, \lambda}^{\left(\beta_{1}, \cdots, \beta_{r} ; \beta\right)}\left(x_{1} \cdots x_{r} ; z, u\right) .
$$

and

$$
\begin{gathered}
{ }_{H} G_{n, \lambda}^{\left(\alpha_{1}+\beta_{1}, \cdots, \alpha_{r}+\beta_{r} ; \alpha+\beta\right)}\left(x_{1} \cdots x_{r} ; x+z, y+u\right) \\
=\sum_{l=0}^{n} H_{l, \lambda} G^{\left(\alpha_{1}, \cdots, \alpha_{r} ; \alpha\right)}\left(x_{1} \cdots x_{r} ; x, y\right)_{H} G_{n-l, \lambda}^{\left(\beta_{1}, \cdots, \beta_{r} ; \beta\right)}\left(x_{1} \cdots x_{r} ; z, u\right) .
\end{gathered}
$$

3. Summation formulae for generalized Lagrange-based Apostol-type Hermite polynomials

First, we prove the following result involving generalized Lagrange-based Apostoltype Hermite polynomials by using series rearrangement techniques. We now begin with the following theorem.

Theorem 3.1. The following implicit summation formulae for Lagrange-based Apostol type Hermite polynomials $T_{n, \lambda, k}^{\left(\alpha_{1}, \cdots, \alpha_{r} ; \alpha\right)}\left(x_{1} \cdots x_{r} ; x, y\right)$ holds true:

$T_{n, \lambda, k}^{\left(\alpha_{1}, \cdots, \alpha_{r} ; \alpha\right)}\left(x_{1} \cdots x_{r} ; x, y\right)=\sum_{m=0}^{n} T_{m, \lambda, k}^{\left(\alpha_{1}, \cdots, \alpha_{r} ; \alpha\right)}\left(x_{1} \cdots x_{r} ; x-z\right) t^{m} H_{n-m}(z, y) \frac{1}{(n-m) !}$.

Proof. By the exploiting generating function (2.1) and using the (1.10)

$$
\begin{aligned}
& \prod_{j=1}^{r}\left(1-x_{j} t\right)^{-\alpha_{j}}\left(\frac{2^{k} t}{\lambda e^{t}+(-1)^{k+1}}\right)^{\alpha} e^{(x-z) t} e^{z t+y t^{2}} \\
= & \sum_{m=0}^{\infty} T_{m, \lambda, k}^{\left(\alpha_{1}, \cdots, \alpha_{r} ; \alpha\right)}\left(x_{1} \cdots x_{r} ; x-z\right) t^{m} \sum_{n=0}^{\infty} H_{n}(z, y) \frac{t^{n}}{n !} .
\end{aligned}
$$

Now replacing $n$ by $n-m$ and comparing the coefficients of $t^{n}$, we get the result (3.1).

Theorem 3.2. The following implicit summation formulae for Lagrange-based Apostol type Hermite polynomials $T_{n, \lambda, k}^{\left(\alpha_{1}, \cdots, \alpha_{r} ; \alpha\right)}\left(x_{1} \cdots x_{r} ; x, y\right)$ holds true:

$T_{n, \lambda, k}^{\left(\alpha_{1}, \cdots, \alpha_{r} ; \alpha\right)}\left(x_{1} \cdots x_{r} ; x+u, y+w\right)=\sum_{m=0}^{n} T_{m, \lambda, k}^{\left(\alpha_{1}, \cdots, \alpha_{r} ; \alpha\right)}\left(x_{1} \cdots x_{r} ; x-z\right) t^{m} H_{n-m}(u, w) \frac{1}{(n-m) !}$.

Proof. Applying the definition (2.1), we have

$$
\begin{gathered}
\prod_{j=1}^{r}\left(1-x_{j} t\right)^{-\alpha_{j}}\left(\frac{2^{k} t}{\lambda e^{t}+(-1)^{k+1}}\right)^{\alpha} e^{(x+u) t+(y+w) t^{2}}=\sum_{n=0}^{\infty} T_{n, \lambda, k}^{\left(\alpha_{1}, \cdots, \alpha_{r} ; \alpha\right)}\left(x_{1} \cdots x_{r} ; x+u, y+w\right) t^{n} \\
=\sum_{m=0}^{\infty} T_{m, \lambda, k}^{\left(\alpha_{1}, \cdots, \alpha_{r} ; \alpha\right)}\left(x_{1} \cdots x_{r} ; x, y\right) t^{m} \sum_{n=0}^{\infty} H_{n}(u, w) \frac{t^{n}}{n !} .
\end{gathered}
$$

Replacing $n$ by $n-m$ in the r.h.s and comparing the coefficient of $t^{n}$, we get the result (3.2).

Theorem 3.3. The following implicit summation formulae for Lagrange-based Apostol type Hermite polynomials $T_{n, \lambda, k}^{\left(\alpha_{1}, \cdots, \alpha_{r} ; \alpha\right)}\left(x_{1} \cdots x_{r} ; x, y\right)$ holds true: 


$$
T_{n, \lambda, k}^{\left(\alpha_{1}, \cdots, \alpha_{r} ; \alpha\right)}\left(x_{1} \cdots x_{r} ; x, y\right)=\sum_{m=0}^{n-2 j} \sum_{j=0}^{\left[\frac{n}{2}\right]} T_{m, \lambda, k}^{\left(\alpha_{1}, \cdots, \alpha_{r} ; \alpha\right)} x^{n-m-2 j} y^{j} \frac{1}{(n-m-2 j) !} .
$$

Proof. Using the definition (2.1), we have

$$
\begin{gathered}
\sum_{n=0}^{\infty} T_{n, \lambda, k}^{\left(\alpha_{1}, \cdots, \alpha_{r} ; \alpha\right)}\left(x_{1} \cdots x_{r} ; x, y\right) t^{n}=\prod_{j=1}^{r}\left(1-x_{j} t\right)^{-\alpha_{j}}\left(\frac{2^{k} t}{\lambda e^{t}+(-1)^{k+1}}\right)^{\alpha} e^{x t+y t^{2}} \\
=\sum_{m=0}^{\infty} T_{m, \lambda, k}^{\left(\alpha_{1}, \cdots, \alpha_{r} ; \alpha\right)} t^{n} \sum_{n=0}^{\infty} x^{n} \frac{t^{n}}{n !} \sum_{j=0}^{\infty} y^{j} \frac{t^{2 j}}{j !} \\
=\sum_{n=0}^{\infty} \sum_{m=0}^{n} T_{m, \lambda, k}^{\left(\alpha_{1}, \cdots, \alpha_{r} ; \alpha\right)} x^{n-m} \frac{t^{n}}{(n-m) !} \sum_{j=0}^{\infty} y^{j} \frac{t^{2 j}}{j !}
\end{gathered}
$$

Replacing $n$ by $n-2 j$ in above equation, we get

$$
=\sum_{n=0}^{\infty} \sum_{m=0}^{n-2 j} \sum_{j=0}^{\left[\frac{n}{2}\right]} T_{m, \lambda, k}^{\left(\alpha_{1}, \cdots, \alpha_{r} ; \alpha\right)} x^{n-m-2 j} y^{j} \frac{t^{n}}{(n-m-2 j) !} .
$$

Comparing the coefficient of $t^{n}$, we get the result (3.3).

Theorem 3.4. The following implicit summation formulae for Lagrange-based Apostol type Hermite polynomials $T_{n, \lambda, k}^{\left(\alpha_{1}, \cdots, \alpha_{r} ; \alpha\right)}\left(x_{1} \cdots x_{r} ; x, y\right)$ holds true:

$$
T_{n, \lambda, k}^{\left(\alpha_{1}, \cdots, \alpha_{r} ; \alpha\right)}\left(x_{1} \cdots x_{r} ; x+1, y\right)=\sum_{m=0}^{n} T_{m, \lambda, k}^{\left(\alpha_{1}, \cdots, \alpha_{r} ; \alpha\right)}\left(x_{1} \cdots x_{r} ; x, y\right) t^{m} \frac{1}{(n-m) !} .
$$

Proof. By the definition of generalized Lagrange-based Apostol type polynomials, we have

$$
\begin{gathered}
\sum_{n=0}^{\infty} T_{n, \lambda, k}^{\left(\alpha_{1}, \cdots, \alpha_{r} ; \alpha\right)}\left(x_{1} \cdots x_{r} ; x+1, y\right) t^{n}-\sum_{n=0}^{\infty} T_{n, \lambda, k}^{\left(\alpha_{1}, \cdots, \alpha_{r} ; \alpha\right)}\left(x_{1} \cdots x_{r} ; x, y\right) t^{n} \\
=\prod_{j=1}^{r}\left(1-x_{j} t\right)^{-\alpha_{j}}\left(\frac{2^{k} t}{\lambda e^{t}+(-1)^{k+1}}\right)^{\alpha} e^{x t+y t^{2}}\left(e^{t}-1\right) \\
=\sum_{m=0}^{\infty} T_{m, \lambda, k}^{\left(\alpha_{1}, \cdots, \alpha_{r} ; \alpha\right)}\left(x_{1} \cdots x_{r} ; x, y\right) t^{m} \sum_{n=0}^{\infty} \frac{t^{n}}{n !}-\sum_{n=0}^{\infty} T_{n, \lambda, k}^{\left(\alpha_{1}, \cdots, \alpha_{r} ; \alpha\right)}\left(x_{1} \cdots x_{r} ; x, y\right) t^{n} .
\end{gathered}
$$

Replacing $n$ by $n-m$, we have

$=\sum_{n=0}^{\infty} \sum_{m=0}^{n} T_{m, \lambda, k}^{\left(\alpha_{1}, \cdots, \alpha_{r} ; \alpha\right)}\left(x_{1} \cdots x_{r} ; x, y\right) t^{m} \frac{t^{n}}{(n-m) !}-\sum_{n=0}^{\infty} T_{n, \lambda, k}^{\left(\alpha_{1}, \cdots, \alpha_{r} ; \alpha\right)}\left(x_{1} \cdots x_{r} ; x, y\right) t^{n}$.

Equating their coefficients of $t^{n}$ leads to formula (3.4).

\section{General symmetry identities}

In this section, we establish general symmetry identities for the generalized Lagrangebased Apostol type Hermite polynomials $T_{n, \lambda, k}^{\left(\alpha_{1}, \cdots, \alpha_{r} ; \alpha\right)}\left(x_{1} \cdots x_{r} ; x, y\right)$ and generalized Lagrange-based Apostol type polynomials $T_{n, \lambda, k}^{\left(\alpha_{1}, \cdots, \alpha_{r} ; \alpha\right)}\left(x_{1} \cdots x_{r} ; x\right)$ by applying the generating functions (2.1) and (1.6). Such type of works, introduced here by the approach given in the recent works of Khan [5] and Pathan and Khan [13, 14, 15]. 
Theorem 4.1. Let $a, b>0$ and $a \neq b$. Then for $x, y \in \mathbb{R}$ and $n \geq 0$, the following identity holds true:

$$
\begin{aligned}
& \sum_{m=0}^{n} a^{n-m} b^{m} T_{n-m, \lambda, k}^{\left(\alpha_{1}, \cdots, \alpha_{r} ; \alpha\right)}\left(x_{1} \cdots x_{r} ; b x, b^{2} y\right) T_{m, \lambda, k}^{\left(\alpha_{1}, \cdots, \alpha_{r} ; \alpha\right)}\left(x_{1} \cdots x_{r} ; a x, a^{2} y\right) \\
= & \sum_{m=0}^{n} b^{n-m} a^{m} T_{n-m, \lambda, k}^{\left(\alpha_{1}, \cdots, \alpha_{r} ; \alpha\right)}\left(x_{1} \cdots x_{r} ; a x, a^{2} y\right) T_{m, \lambda, k}^{\left(\alpha_{1}, \cdots, \alpha_{r} ; \alpha\right)}\left(x_{1} \cdots x_{r} ; b x, b^{2} y\right) .
\end{aligned}
$$

Proof. Start with

$$
G(t)=\prod_{j=1}^{r}\left(1-x_{j} a b t\right)^{-\alpha_{j}}\left(\frac{2^{2 k} a b t}{\left(\lambda e^{a t}+(-1)^{k+1}\right)\left(\lambda e^{b t}+(-1)^{k+1}\right)}\right)^{\alpha} e^{a b x t+a^{2} b^{2} y t^{2}} .
$$

Then the expression for $G(t)$ is symmetric in $a$ and $b$ and we can expand $G(t)$ into series in two ways to obtain:

$$
\begin{gathered}
G(t)=\sum_{n=0}^{\infty} T_{n, \lambda, k}^{\left(\alpha_{1}, \cdots, \alpha_{r} ; \alpha\right)}\left(x_{1} \cdots x_{r} ; b x, b^{2} y\right)(a t)^{n} \sum_{m=0}^{\infty} T_{m, \lambda, k}^{\left(\alpha_{1}, \cdots, \alpha_{r} ; \alpha\right)}\left(x_{1} \cdots x_{r} ; a x, a^{2} y\right)(b t)^{m} \\
=\sum_{n=0}^{\infty} \sum_{m=0}^{n} a^{n-m} b^{m} T_{n-m, \lambda, k}^{\left(\alpha_{1}, \cdots, \alpha_{r} ; \alpha\right)}\left(x_{1} \cdots x_{r} ; b x, b^{2} y\right) T_{m, \lambda, k}^{\left(\alpha_{1}, \cdots, \alpha_{r} ; \alpha\right)}\left(x_{1} \cdots x_{r} ; a x, a^{2} y\right) t^{n} .
\end{gathered}
$$

On the similar lines we can show that

$$
\begin{aligned}
& G(t)=\sum_{n=0}^{\infty} T_{n, \lambda, k}^{\left(\alpha_{1}, \cdots, \alpha_{r} ; \alpha\right)}\left(x_{1} \cdots x_{r} ; a x, a^{2} y\right)(a t)^{n} \sum_{m=0}^{\infty} T_{m, \lambda, k}^{\left(\alpha_{1}, \cdots, \alpha_{r} ; \alpha\right)}\left(x_{1} \cdots x_{r} ; b x, b^{2} y\right)(b t)^{m} \\
& =\sum_{n=0}^{\infty} \sum_{m=0}^{n} b^{n-m} a^{m} T_{n-m, \lambda, k}^{\left(\alpha_{1}, \cdots, \alpha_{r} ; \alpha\right)}\left(x_{1} \cdots x_{r} ; a x, a^{2} y\right) T_{m, \lambda, k}^{\left(\alpha_{1}, \cdots, \alpha_{r} ; \alpha\right)}\left(x_{1} \cdots x_{r} ; b x, b^{2} y\right) t^{n} .
\end{aligned}
$$

By comparing the coefficients of $t^{n}$ on the right hand sides of the last two equations, we arrive the desired result.

Remark 4.1. For $\alpha=1$, the above result reduces to

$$
\begin{aligned}
& \sum_{m=0}^{n} a^{n-m} b^{m} T_{n-m, \lambda, k}^{\left(\alpha_{1}, \cdots, \alpha_{r}\right)}\left(x_{1} \cdots x_{r} ; b x, b^{2} y\right) T_{m, \lambda, k}^{\left(\alpha_{1}, \cdots, \alpha_{r}\right)}\left(x_{1} \cdots x_{r} ; a x, a^{2} y\right) \\
= & \sum_{m=0}^{n} b^{n-m} a^{m} T_{n-m, \lambda, k}^{\left(\alpha_{1}, \cdots, \alpha_{r}\right)}\left(x_{1} \cdots x_{r} ; a x, a^{2} y\right) T_{m, \lambda, k}^{\left(\alpha_{1}, \cdots, \alpha_{r}\right)}\left(x_{1} \cdots x_{r} ; b x, b^{2} y\right) .
\end{aligned}
$$

Remark 4.2. By setting $b=1$ in Theorem 4.1, we immediately get the following result

$$
\begin{aligned}
& \sum_{m=0}^{n} a^{n-m} T_{n-m, \lambda, k}^{\left(\alpha_{1}, \cdots, \alpha_{r} ; \alpha\right)}\left(x_{1} \cdots x_{r} ; x, y\right) T_{m, \lambda, k}^{\left(\alpha_{1}, \cdots, \alpha_{r} ; \alpha\right)}\left(x_{1} \cdots x_{r} ; a x, a^{2} y\right) \\
= & \sum_{m=0}^{n} a^{m} T_{n-m, \lambda, k}^{\left(\alpha_{1}, \cdots, \alpha_{r} ; \alpha\right)}\left(x_{1} \cdots x_{r} ; a x, a^{2} y\right) T_{m, \lambda, k}^{\left(\alpha_{1}, \cdots, \alpha_{r} ; \alpha\right)}\left(x_{1} \cdots x_{r} ; x, y\right) .
\end{aligned}
$$

Theorem 4.2. Let $a, b, c>0$ and $a \neq b$. Then for $x, y \in \mathbb{R}$ and $n \geq 0$, the following identity holds true:

$$
\sum_{m=0}^{n} \sum_{i=0}^{a-1} \sum_{j=0}^{b-1}(-\lambda)^{i+j} a^{n-m} b^{m} T_{n, \lambda, k}^{\left(\alpha_{1}, \cdots, \alpha_{r}, \alpha\right)}\left(b x+\frac{b}{a} i+j, b^{2} z\right) T_{m, \lambda, k}^{\left(\alpha_{1}, \cdots, \alpha_{r}, \alpha\right)}(a y)
$$


$=\sum_{m=0}^{n} \sum_{i=0}^{b-1} \sum_{j=0}^{a-1}(-\lambda)^{i+j} a^{m} b^{n-m} T_{n, \lambda, k}^{\left(\alpha_{1}, \cdots, \alpha_{r}, \alpha\right)}\left(a x+\frac{a}{b} i+j, a^{2} z\right) T_{m, \lambda, k}^{\left(\alpha_{1}, \cdots, \alpha_{r}, \alpha\right)}(b y)$.

Proof. Let

$$
\begin{aligned}
& H(t)=\prod_{j=1}^{r}\left(1-x_{j} a b t\right)^{-\alpha_{j}}\left(\frac{2^{2 k} a b t^{2}}{\left(\lambda e^{a t}+(-1)^{k+1}\right)\left(\lambda e^{b t}+(-1)^{k+1}\right)}\right)^{\alpha} \frac{1+\lambda(-1)^{a+1} e^{a b t}}{\left(\lambda e^{a t}+1\right)\left(\lambda e^{b t}+1\right)} e^{a b(x+y) t+a^{2} b^{2} z t^{2}} . \\
& g(t)=\prod_{j=1}^{r}\left(1-x_{j} a t\right)^{-\alpha_{j}}\left(\frac{2^{k} a t}{\left(\lambda e^{a t}+(-1)^{k+1}\right)}\right)^{\alpha} e^{a b x t+a^{2} b^{2} z t^{2}}\left(\frac{1-\lambda\left(e^{-b t}\right)^{a}}{\lambda e^{b t}+1}\right) \\
& \times \prod_{j=1}^{r}\left(1-x_{j} b t\right)^{-\alpha_{j}}\left(\frac{2^{k} b t}{\left(\lambda e^{b t}+(-1)^{k+1}\right)}\right)^{\alpha} e^{a b y t}\left(\frac{1-\lambda\left(e^{-a t}\right)^{b}}{\lambda e^{a t}+1}\right) \\
& =\prod_{j=1}^{r}\left(1-x_{j} a t\right)^{-\alpha_{j}}\left(\frac{2^{k} a t}{\left(\lambda e^{a t}+(-1)^{k+1}\right)}\right)^{\alpha} e^{a b x t+a^{2} b^{2} z t^{2}} \sum_{i=0}^{a-1}(-\lambda)^{i}\left(e^{b t i}\right) \\
& \times \prod_{j=1}^{r}\left(1-x_{j} b t\right)^{-\alpha_{j}}\left(\frac{2^{k} b t}{\left(\lambda e^{b t}+(-1)^{k+1}\right)}\right)^{\alpha} e^{a b y t} \sum_{j=0}^{b-1}(-\lambda)^{j}\left(e^{a t j}\right) \\
& =\prod_{j=1}^{r}\left(1-x_{j} a t\right)^{-\alpha_{j}}\left(\frac{2^{k} a t}{\left(\lambda e^{a t}+(-1)^{k+1}\right)}\right)^{\alpha} e^{a^{2} b^{2} z t^{2}} \sum_{i=0}^{a-1} \sum_{j=0}^{b-1}(-\lambda)^{i+j} e^{\left(b x+\frac{b}{a} i+j\right) a t} \\
& \times \sum_{m=0}^{\infty} T_{m, \lambda, k}^{\left(\alpha_{1}, \cdots, \alpha_{r}, \alpha\right)}(a y)(b t)^{m} . \\
& =\sum_{n=0}^{\infty} \sum_{i=0}^{a-1} \sum_{j=0}^{b-1}(-\lambda)^{i+j} T_{n, \lambda, k}^{\left(\alpha_{1}, \cdots, \alpha_{r}, \alpha\right)}\left(b x+\frac{b}{a} i+j, b^{2} z\right)(a t)^{n} \sum_{m=0}^{\infty} T_{m, \lambda, k}^{\left(\alpha_{1}, \cdots, \alpha_{r}, \alpha\right)}(a y)(b t)^{m} \\
& =\sum_{n=0}^{\infty} \sum_{m=0}^{n} \sum_{i=0}^{a-1} \sum_{j=0}^{b-1}(-\lambda)^{i+j} a^{n-m} b^{m} T_{n, \lambda, k}^{\left(\alpha_{1}, \cdots, \alpha_{r}, \alpha\right)}\left(b x+\frac{b}{a} i+j, b^{2} z\right) T_{m, \lambda, k}^{\left(\alpha_{1}, \cdots, \alpha_{r}, \alpha\right)}(a y) t^{n} .
\end{aligned}
$$

Since $(-1)^{a+1}=(-1)^{b+1}$, the expression for

$$
H(t)=\prod_{j=1}^{r}\left(1-x_{j} a b t\right)^{-\alpha_{j}}\left(\frac{2^{2 k} a b t^{2}}{\left(\lambda e^{a t}+(-1)^{k+1}\right)\left(\lambda e^{b t}+(-1)^{k+1}\right)}\right)^{\alpha} \frac{1+\lambda(-1)^{a+1} e^{a b t}}{\left(\lambda e^{a t}+1\right)\left(\lambda e^{b t}+1\right)} e^{a b(x+y) t+a^{2} b^{2} z t^{2}} .
$$

is symmetric in $a$ and $b$. Therefore, by symmetry we obtain the following power series expansion for $H(t)$

$=\sum_{n=0}^{\infty} \sum_{m=0}^{n} \sum_{i=0}^{b-1} \sum_{j=0}^{a-1}(-\lambda)^{i+j} a^{m} b^{n-m} T_{n, \lambda, k}^{\left(\alpha_{1}, \cdots, \alpha_{r}, \alpha\right)}\left(a x+\frac{a}{b} i+j, a^{2} z\right) T_{m, \lambda, k}^{\left(\alpha_{1}, \cdots, \alpha_{r}, \alpha\right)}(b y) t^{n}$.

By comparing the coefficients of $t^{n}$ on the right hand sides of the last two equations, we arrive at the desired result.

\section{References}

[1] Andrews, L. C, Special functions for engineers and mathematicians, Macmillan Co. New York, 1985.

[2] Bell, E. T, Exponential polynomials, Ann. of Math., 35(1934), 258-277. 
[3] Chan, C. W.-Ch, Chyan, Ch. J and Srivastava, H. M, The Lagrange polynomials in severa variables, Integ. Trans. Spec. Funct., 12(2001), 139-148.

[4] Dattoli, G, Lorenzutta, S and Cesarano, C, Finite sums and generalized forms of Bernoulli polynomials, Rendiconti di Mathematica, 19(1999), 385-391.

[5] Khan, W. A: Some properties of the generalized Apostol type Hermite-based polynomials, Kyungpook Math. J., 55(2015), 597-614.

[6] Lu, D. Q and Luo, Q. M, Some properties of the generalized Apostol tpe polynomials, Boundary Value Problems., (2013), 2013:64.

[7] Luo, Q. M, Apostol Euler polynomials of higher order and gaussian hypergeometric functions, Taiwanese J. Math., 10(4)(2006), 917-925.

[8] Luo, Q. M, q-extensions for the Apostol-Genocchi polynomials, Gen. Math., $17(2)(2009), 113-125$.

[9] Luo, Q. M, Extensions for the Genocchi polynomials and its fourier expansions and integral representations, Osaka J. Math., 48(2011), 291-310.

[10] Luo, Q. M and Srivastava, H. M, Some generalizations of the Apostol-Bernoulli and Apostol-Euler polynomials, J. Math. Anal. Appl., 308(1)(2005), 290-302.

[11] Luo, Q. M and Srivastava, H. M, Some generalizations of the Apostol Genocchi polynomials and the Stirling number of the second kind, Appl. Math. Comput., 217(2011), 5702-5728.

[12] Luo, Q. M and Srivastava, H. M, Some relationships between the ApostolBernoulli and Apostol-Euler polynomials, Comput. Math. Appl., 51(2006), 631-642.

[13] Pathan, M. A and Khan, W. A, Some implicit summation formulas and symmetric identities for the generalized Hermite-based polynomials, Acta Universitatis Apulensis, 39(2014), 113-136.

[14] Pathan, M. A and Khan, W. A, Some implicit summation formulas and symmetric identities for the generalized Hermite-Bernoulli polynomials, Mediterr. J. Math., 12 (2015), 679-695.

[15] Pathan, M. A and Khan, W. A, A new class of generalized polynomials associated with Hermite and Euler polynomials, Mediterr. J. Math., 13(2016), 913-928 .

[16] Srivastava, H. M, Ozarslan, M. A, Kaanoglu, C, Some generalized Lagrangebased Apostol Bernoulli, Apostol Euler and Apostol Genocchi polynomials, Russian J. Math. Phy., 20(2013), 110-120. 